\title{
Aposentadoria, saúde do idoso e saúde do trabalhador: Revisão integrativa da produção brasileira
}

Retirement, elderly health, and worker health: An integrative review of the brazilian literature Jubilación, salud del anciano y salud del trabajador: Revisión integradora de la producción brasileña

\author{
Marcos Henrique ANTUNES ${ }^{1}$ \\ Carmen Leontina Ojeda Ocampo MORÉ \\ Universidade Federal de Santa Catarina, Florianópolis, SC, Brasil
}

Resumo Este estudo de revisão integrativa teve como objetivo analisar a produção científica brasileira sobre aposentadoria, saúde do idoso e saúde do trabalhador. Realizou-se uma busca nas bases de dados SciELO e LILACS acerca de artigos publicados no período entre 2004 e maio de 2015. Os descritores utilizados foram aposentadoria, saúde do idoso e saúde do trabalhador, a partir dos quais foram localizadas 82 produções. Após verificação dos critérios de inclusão e exclusão previamente estabelecidos, foram selecionados e analisados 20 artigos. Os resultados mostraram a predominância de estudos que versam sobre a interface entre aposentadoria e saúde do idoso, indicando a carência de publicações que explorem a relação entre aposentadoria e saúde do trabalhador, bem como entre saúde do idoso e saúde do trabalhador. Ressalta-se a necessidade de produções que tratem sobre tais temas, possibilitando uma melhor compreensão da relação entre os mesmos e subsidiando políticas e ações direcionadas para esses públicos em diferentes espaços sociais, dentre os quais se situa o contexto organizacional e do trabalho.

Palavras-chave:

Aposentadoria; saúde do idoso; saúde do trabalhador.

Abstract This study aimed at presenting an integrated review of the Brazilian scientific production on the issues of retirement, elderly health, and worker health. The SciELO and LILACS data bases were searched for articles published between 2004 and May 2015. Search keywords used were retirement, elderly health, and worker health, for which 82 articles were found. After verifying the previously established inclusion and exclusion criteria, 20 articles were selected for analysis. Results showed a predominance of studies discussing the interface between retirement and elderly health, suggesting a lack of publications exploring the relation between retirement and worker health, as well as between elderly and worker health. The need is highlighted for publications dealing with these issues, enabling a better understanding of the relationship between these issues, fostering policies and actions directed towards this population in different social spheres, within which lie the contexts of work and organization.

Keywords:

Retirement; elderly health; worker health.

Resumen Este estudio de revisión integradora tuvo como objetivo analizar la producción científica brasileña acerca de la jubilación, la salud del anciano y la salud del trabajador. Se realizó una búsqueda en las bases de datos SciELO y LILACS respecto de los artículos publicados entre 2004 y mayo de 2015. Los descriptores utilizados fueron jubilación, salud del anciano y salud del trabajador, a partir de los cuales se localizaron 82 producciones. Tras revisar los criterios de inclusión y exclusión establecidos previamente, se seleccionaron y

Endereço para correspondência: Rua Capitão Romualdo de Barros, 611, Carvoeira, Florianópolis, SC, Brasil 88040-600. Email: marcos.antunes@live.com 
se analizaron 20 artículos. Los resultados mostraron un predominio de los estudios que se ocupan de la interfaz entre jubilación y salud del anciano, lo que indica una carencia de publicaciones que exploren la relación entre la jubilación y la salud del trabajadore, así como entre la salud del anciano y la salud del trabajador. Se enfatiza la necesidad de producciones direccionadas a estos temas, permitiendo una mejor comprensión entre ellos y subsidiando políticas y acciones dirigidas a estos públicos en diferentes espacios sociales, entre los que se encuentra el contexto de la organización y del trabajo.

Palabras-clave:

Jubilación; salud del anciano; salud del trabajador.

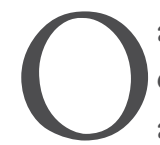

aumento da expectativa de vida é um fenômeno mundial. Nos países em desenvolvimento, como é o caso do Brasil, esse processo tem ocorrido de forma ainda mais acelerada em decorrência do acesso aos serviços de saúde, dos avanços da ciência, e, consequentemente, do aumento da qualidade de vida. Dados do IBGE (2011) acerca do último censo demográfico nacional evidenciam que, em um período de 10 anos, houve uma mudança significativa da população do país, com a diminuição da taxa de natalidade e o acréscimo do nível de longevidade. Diante dessa realidade, torna-se cada vez mais comum a presença de aposentados, idosos e longevos nos espaços sociais, sendo que esses públicos demandam especial atenção na contemporaneidade, especialmente, sob a perspectiva da saúde.

Esse contingente populacional constitui-se em um grupo heterogêneo e quantitativamente significativo no país, cujas características devem ser entendidas à luz de sua singularidade, tendo em vista que o envelhecimento é um processo de franca mudança e que ocorre ao longo de toda a vida. Assim, pensar sobre o envelhecimento exige analisar as tensões entre os processos biológicos e psicossociais de vida, atentando para as particularidades da história de cada indivíduo e do contexto no qual ele se insere, em uma perspectiva capaz de integrar as diferentes experiências ao longo do curso de vida, sem estigmas e/ou hierarquias entre elas (Brasil, 2014; Mendes, Gusmão, Faro, \& Leite, 2005).

Em certa medida, esse movimento coloca uma ruptura com a forma pela qual, historicamente, o envelhecimento e a pessoa idosa foram entendidos. Dessa maneira, considerando a funcionalidade da pessoa idosa e em uma perspectiva de saúde ampliada, que vai além da prevenção de doenças, aponta-se para uma compreensão que contemple principalmente a inter-relação de aspectos físicos, mentais e a existência de redes de suporte social e promoção de autonomia (Lei no 8.842/1994). Percebe-se, inclusive, que há um esforço para o avanço na construção de políticas públicas e efetivação dos direitos para essa população. No campo da saúde, por exemplo, tais políticas passaram a apontar diretrizes técnicas para atendimento global de sujeitos nas fases tardias da vida, envolvendo prevenção, promoção e recuperação da saúde, com vistas à melhoria da qualidade de vida e autonomia. Há, ainda, a iniciativa de implementar espaços para estimular a participação dos idosos em suas comunidades, dentre os quais estão os centros de convivência, que oportunizam o acesso a práticas de atividades físicas, culturais, educativas, sociais e de lazer (Brasil, 2006; Neri \& Freire, 2000).

No que diz respeito à aposentadoria, para além de um direito previdenciário, ela se configura como um dos principais eventos críticos da vida adulta, abrangendo diversos processos psicológicos e sociais (Antunes, Soares, \& Silva, 2015). Como menciona Santos (1990), um dos conflitos que acontecem nesse período pode ser caracterizado pela dualidade crise versus liberdade. Assim, por um lado, a aposentadoria é vivenciada com tensão e dificuldade de adaptação ao novo momento e, por outro lado, pode ser sinônimo de liberdade e potencialização das fontes de satisfação devido ao maior tempo livre após o desligamento do trabalho. Diferentes estudos na área (Denton \& Spencer, 2009; França, 2014a; Soares \& Costa, 2011; Zanelli, Silva, \& Soares, 2010) demonstram que os aspectos que balizam esse conflito estão interligados à história de vida do indivíduo, permeada pelo modo como construiu sua identidade, suas relações familiares, de amizade, de trabalho e sua trajetória profissional.

Em virtude das transformações demográficas, nota-se que nas últimas décadas houve um progresso significativo na produção científica sobre a aposentadoria, seja no contexto nacional ou internacional (França \& Soares, 2009; Johnson, 2009; Szinovacz, Ekerdt, Butt, Barton, \& Oala, 2012). Isso é particularmente importante se observada a necessidade de estudos que ampliem a compreensão do conjunto de aspectos relacionados ao fenômeno, de modo a ultrapassar, principalmente, a visão do aposentado como "velhinho" e que deve usar pijamas, conforme salientou Rodrigues (2001). 
Estudos recentes de revisão da literatura mostram algumas características das publicações relacionadas ao tema. Em uma revisão integrativa da produção brasileira sobre família, trabalho e aposentadoria, Antunes e Moré (2014) averiguaram a predominância de pesquisas que relacionam a aposentadoria ao trabalho, se comparadas, por exemplo, as que pautam a aposentadoria interligada à família. Esse fato justifica-se, possivelmente, pela natureza do fenômeno da aposentadoria, que se origina e está intrinsecamente vinculada à dimensão do mundo do trabalho. Todavia, problematiza-se essa questão, visto que ela pode limitar a discussão do tema, desconsiderando um fator de grande importância na vivência e adaptação ao desligamento laboral, que é o contexto familiar.

Já a revisão sobre aposentadoria e saúde mental, realizada por Panozzo e Monteiro (2013), identificou que há carência de estudos que se detenham a discutir aspectos psicológicos e da relação entre envelhecimento e aposentadoria. Essa revisão também apontou que a aposentadoria ainda é consideravelmente pensada sob a ótica econômica, sendo esse, evidentemente, um aspecto saliente do processo, mas que não é capaz de abarcar a complexidade de fatores imbricados em tal experiência.

Ao abordar a temática da aposentadoria, Cockell (2014) aponta que essa questão é um dos grandes desafios para a área da saúde no Brasil, pois as desigualdades sociais tornam os idosos ainda mais vulneráveis diante do modelo atual de trabalho, ocasionando repercussões em sua saúde, nas relações estabelecidas no mundo laboral e na família, assim como na manutenção do sistema previdenciário vigente. Há, portanto, a inter-relação de diferentes aspectos que estão envolvidos no debate, de modo que as ações não devem ser pensadas de forma pontual, mas buscando viabilizar o diálogo acerca dos diferentes temas.

França (2014b) refere que a promoção de saúde das pessoas em transição para aposentadoria deve estar fundamentada em um modelo integrativo de prevenção ao surgimento de transtornos e de promoção de competências, levando-se em conta os recursos de ordem individual, psicossocial e organizacional. Nessa perspectiva, vale destacar que existem dispositivos legais que preveem a execução de programas de orientação para aposentadoria, os quais se configuram como ações de promoção e prevenção de saúde. São eles: o Plano de Ação Internacional para o Envelhecimento (ONU, 2002) e o Estatuto do Idoso (Lei no 10.741/2003). Ambos os documentos apontam que tais programas de desenvolvimento podem contribuir para que os trabalhadores alcancem um envelhecimento com saúde e bem-estar. Ainda nessa linha, dentro da Política Nacional do Idoso (Lei no 8.842/1994) e da Política de Atenção à Saúde e Segurança do Trabalho do Servidor Público Federal (PASS) são abordados aspectos em relação ao afastamento das atividades laborais, chamando a atenção para as práticas de gestão nas organizações, sobretudo em relação à prevenção de riscos e doenças, ao envelhecimento ativo e à preparação para a aposentadoria. Assim, percebe-se que a discussão acerca da aposentadoria pode se localizar entre os campos de saúde do idoso e saúde do trabalhador, tendo em vista que a vivência desse período não ocorre de forma isolada, mas se interliga, entre outros fatores, à sua trajetória profissional e às diferentes etapas que compõem o ciclo de vida.

Com isso em vista, este estudo foi desenvolvido com o objetivo de analisar a produção científica brasileira sobre aposentadoria, saúde do idoso e saúde do trabalhador na última década. Por meio da revisão integrativa dessas temáticas, pretende-se distinguir o estado do conhecimento e contribuir para a execução de futuras pesquisas. Acredita-se que este trabalho pode auxiliar na verificação da importância do debate na intersecção das temáticas em questão, ampliando o foco de discussão em torno das mesmas.

\section{MÉTODO}

Este estudo se afigura como uma revisão integrativa da literatura, uma vez que organiza e sumariza pesquisas passadas, além de realizar apontamentos acerca de um corpo específico da produção científica. Esse tipo de revisão contribui na ampliação da discussão sobre determinado assunto, pois possibilita reunir e analisar, a partir de critérios estabelecidos, os resultados obtidos com investigações em certos temas e/ou áreas, e, a partir disso, oferecer sugestões para a realização de futuras pesquisas (Beyea \& Nicoll, 1998; Mendes, Silveira, \& Galvão, 2008).

Este trabalho apresenta os resultados identificados a partir da busca de artigos nacionais que versam sobre aposentadoria, saúde do idoso e saúde do trabalhador e que tenham sido publicados na última década. O levantamento foi realizado na segunda semana do mês de junho de 2015, nas seguintes bases de dados: Scientific Eletronic Library Online (SciELO) e LILACS. A pesquisa ocorreu por meio da combinação de duas palavras-chave, as quais foram intermediadas pelo uso do operador booleano "AND". Dessa maneira, as estratégias de 
busca aplicadas foram: (a) aposentadoria AND saúde do idoso, (b) aposentadoria AND saúde do trabalbador e (c) saúde do idoso AND saúde do trabalhador. Cabe mencionar que, nessa revisão, optou-se por empregar o termo Saúde do idoso em vez de Saúde da pessoa idosa para conferir uniformidade à discussão, tendo em vista que, ao contrário da base LILACS, a SciELO não utiliza o segundo termo como descritor.

Os critérios de inclusão utilizados nessa revisão foram: (a) exclusivamente artigos que apresentassem resultados de investigação científica ou relatos de experiência; (b) unicamente produções desenvolvidas no contexto brasileiro, com o objetivo de verificar quais as publicações veiculadas no país; (c) artigos publicados no período entre 2004 e maio de 2015, afim de reconhecer como tais temas tem sido abordados em investigações recentes, desenvolvidas ao longo da última década, sobretudo, em vista das mudanças demográficas já citadas; e (d) publicações que estivessem disponíveis eletronicamente. Frente a essas delimitações, foram excluídos diversos tipos de trabalho, como teses, dissertações, livros e capítulos de livros, resenhas e ensaios teóricos.

A partir dos resultados obtidos no levantamento, o procedimento para seleção dos artigos que compõe o corpus de análise deste trabalho, consistiu, primeiramente, na leitura das palavras-chave e dos resumos, de modo a priorizar as produções que atendiam aos objetivos definidos, o que culminou na subtração das publicações duplicadas e das que não estavam diretamente ligadas ao tema em questão. Após essa triagem, cada um dos artigos eleitos foi lido na integra e descrito, a fim de registrar suas principais informações. Os dados foram analisados segundo os princípios da análise de conteúdo proposta por Olabuénaga (2009), tendo como referência as seguintes questões norteadoras: (a) quais são as características metodológicas dos estudos (natureza, objetivos e técnicas)? (b) quais são os principais resultados evidenciados nos estudos selecionados em cada uma das três estratégias de busca? Cabe informar, ainda, que a categorização foi estabelecida de acordo com os descritores empregados na busca pelas produções. Isso possibilitou observar como as relações temáticas vêm sendo abordadas e quais os seus principais direcionamentos.

\section{RESULTADOS}

Mediante a pesquisa realizada nas referidas bases de dados, foi localizado um total de 82 produções, sendo que 14 encontravam-se na SciELO e 68 na LILACS. Após a leitura dos resumos desses trabalhos e considerando-se os critérios de inclusão e exclusão, foram deduzidos do resultado inicial: 39 que não preenchiam os critérios estabelecidos, 18 que se encontravam duplicados e 5 que não contemplavam diretamente a discussão dos temas de interesse. Assim, por meio desse processo, foram selecionados 20 artigos que serão analisados ao longo desta revisão.

A apresentação e a discussão dos estudos eleitos encontram-se organizadas em dois segmentos que correspondem às questões norteadoras do processo de análise. O primeiro deles apresenta as características identificadas nessas produções acerca de seu período de publicação e metodologia; o segundo descreve qualitativamente os resultados e as contribuições que tais artigos oferecem para o entendimento do objetivo dessa revisão. Em relação ao primeiro segmento, um aspecto a ser considerado inicialmente é o número de trabalhos encontrados, que indica uma possível restrição da produção científica brasileira sobre aposentadoria, saúde do idoso e saúde do trabalhador. Isso fica mais evidente se analisado frente ao fato de que o levantamento compreendeu um período de 10 anos e meio, e, ainda assim, obteve-se um resultado final de apenas 20 artigos.

Cabe evidenciar, também, a distribuição dos artigos em relação às estratégias de buscas utilizadas. Dos 20 artigos, 12 tratam da relação entre aposentadoria e saúde do idoso, 5 abordam aposentadoria vinculada à saúde do trabalhador e 3 relacionam saúde do idoso e saúde do trabalhador. Os focos mais precisos desses artigos serão discutidos posteriormente, porém, convém destacar a primazia de estudos que analisam a interface entre aposentadoria e saúde do idoso. A Tabela 1 foi desenvolvida com o intuito de sintetizar as principais informações que caracterizam metodologicamente as publicações analisadas.

TABELA 1. Características metodológicas dos artigos

\begin{tabular}{lll}
\multicolumn{1}{c}{ Autor/ano } & Natureza da pesquisa & Técnica de coleta de dados \\
Brant e Gomes (2005) & Qualitativa & Entrevista aberta \\
Trentini, Silva, Valle e Hammerschmidt & Qualitativa & Entrevista aberta \\
$(2005)$ & Qualitativa & Entrevista \\
Lesbaupin e Malerbi (2006) & Quali-quantitativa & Entrevista, diário de campo e questioná- \\
Salimene et al. (2006) & rios
\end{tabular}




\begin{tabular}{|c|c|c|}
\hline Autor/ano & Natureza da pesquisa & Técnica de coleta de dados \\
\hline Cavalcante e Freitas (2007) & Qualitativa & Relato de experiência \\
\hline $\begin{array}{l}\text { Garrido, Paiva, Nascimento, Sousa e } \\
\text { Santos (2007) }\end{array}$ & Qualitativa & Grupo focal \\
\hline Paskulin e Vianna (2007) & Quantitativa & Inquérito \\
\hline Rezende e Dias (2007) & Qualitativa & Entrevista semiestruturada \\
\hline $\begin{array}{l}\text { Miranda, Carvalho, Fernandes, Silva e } \\
\text { Sabino (2009) }\end{array}$ & Quantitativa & $\begin{array}{l}\text { Dados secundários (fichas e boletins da } \\
\text { Instituição) }\end{array}$ \\
\hline $\begin{array}{l}\text { Robazzi, Marziale, Rodrigues, Silveira e } \\
\text { Alves (2009) }\end{array}$ & Quantitativa & Análise documental \\
\hline Andrade, Silva e Santos (2010) & Qualitativa & Entrevista estruturada \\
\hline $\begin{array}{l}\text { Celich, Creutzberg, Goldim e Gomes } \\
\text { (2010) }\end{array}$ & Qualitativa & Entrevista \\
\hline Tavares et al. (2010) & Qualitativa & Entrevista semiestruturada \\
\hline Finazzi-Santos e Siqueira (2011) & Qualitativa & Entrevista \\
\hline Carvalho, Santos, Souza e Souza (2012) & Quantitativa & $\begin{array}{l}\text { Questionário (dados secundários - PAD/ } \\
\text { MG) }\end{array}$ \\
\hline $\begin{array}{l}\text { França, Murta, Negreiros, Pedralho e } \\
\text { Carvalhedo (2013) }\end{array}$ & Quali-quantitativa & Relato de intervenção \\
\hline $\begin{array}{l}\text { Holz, Nunes, Thumé, Lange e Facchini } \\
\text { (2013) }\end{array}$ & Quantitativa & Questionários estruturados \\
\hline Marin et al. (2013) & Quali-quantitativa & Entrevista e escala \\
\hline $\begin{array}{l}\text { Antes, Ribeiro, Schneider, Benedetti e } \\
\text { D'orsi (2014) }\end{array}$ & Quantitativa & $\begin{array}{l}\text { Questionário (dados secundários - Perfil } \\
\text { do Idoso } 2002 \text { e EpiFloripa Idoso 2009) }\end{array}$ \\
\hline Brites, Abreu e Pinto (2014) & Quantitativa & Análise documental \\
\hline
\end{tabular}

Referente ao período de publicação, observou-se que 2007 foi o ano em que houve maior concentração, com quatro artigos publicados. Apesar disso, não foi verificada uma tendência específica a alguma parcela do tempo analisado, estando os artigos, portanto, distribuídos ao longo da década. Em relação ao tipo de pesquisa, notou-se o predomínio da abordagem qualitativa, seguida pela quantitativa e, por fim, pela quali-quantitativa. Acerca das técnicas empregadas para coleta de dados, constatou-se um número significativo de estudos realizados a partir de entrevistas, especialmente abertas ou semiestruturadas. Também se sobressaem os estudos de análise documental e com utilização de escalas, inquéritos e questionários.

O segundo segmento de análise, por sua vez, refere-se à discussão de resultados e contribuições dos trabalhos que compõem o corpus deste estudo. Esses elementos estão congregados em três categorias temáticas que foram previamente definidas, tendo como referência as palavras-chave utilizadas como estratégias de busca, sendo elas: aposentadoria e saúde do idoso, aposentadoria e saúde do trabalhador, saúde do idoso e saúde do trabalhador. Assim, na sequência serão descritos os principais aspectos evidenciados em cada um dos artigos, que serão apresentados individualmente com a finalidade de explorar as especificidades dos seus achados e dos contextos nos quais foram desenvolvidos.

\section{Aposentadoria e saúde do idoso}

Foram identificados 12 artigos que abordam a relação entre aposentadoria e saúde do idoso. Dentre eles, 6 direcionam a discussão para as questões relacionadas ao processo de envelhecimento e as condições socioeconômicas e de saúde dos idosos. Lesbaupin e Malerbi (2006) investigam a visão que os idosos têm de si mesmos, sendo possível perceber a tradicional dicotomia com a qual é retratado o processo de envelhecimento: de um lado as perdas e, de outro, os ganhos decorrentes das experiências de vida. Observou-se que as narrativas acerca da aposentadoria denotavam o interesse desses indivíduos de permanecerem ativos e atuando em outros espaços.

Salimene et al. (2006) apresentaram o perfil previdenciário de idosos internados na Clínica Médica de um Hospital Geral da cidade de São Paulo. De 107 pacientes, 55\% eram mulheres e 41\% homens, os quais tinham escolaridade de 3,13 anos e 3,02 anos, respectivamente. No total, 48,6\% recebiam até 1 salário mínimo, 20,5\% recebiam de 1 a 2 salários e $15 \%$ não tinham rendimento mensal. Desses idosos, $44 \%$ eram chefes de família, sendo que 22,5\% eram homens e 21,5\% eram mulheres. Apesar da média de escolaridade das mulheres ser maior que a dos homens, observou-se que elas obtinham rendimentos menores. Ademais, foi averiguado que, 
embora o rendimento mensal dos idosos fosse muito baixo, relataram serem os principais responsáveis pelo sustento de filhos e netos.

Paskulin e Vianna (2007) avaliaram a associação entre sexo e grupo etário com as variáveis socioeconômicas e de saúde em idosos residentes em Porto Alegre (RS). Participaram da pesquisa 292 idosos pertencentes, predominantemente, às classes média e média alta, sendo que 84\% tinham idade entre 60 e 79 anos, 67,8\% eram do sexo feminino e $81 \%$ consideravam-se saudáveis. Desses idosos, $83,6 \%$ recebiam aposentadoria e/ou pensão e $86,6 \%$ não exerciam atividades remuneradas. Os dados evidenciaram que, se comparadas aos homens, as mulheres residiam mais sozinhas, não possuíam companheiro, tinham menor nível de escolaridade, relatavam mais problemas de saúde, faziam maior uso de medicação sistemática, realizavam menos atividades físicas, recebiam menos aposentadoria e exerciam menos atividades remuneradas. Por outro lado, elas percebiam maior apoio das redes sociais informais e consumiam menos bebidas alcoólicas e cigarro.

O estudo de Carvalho et al. (2012), buscou avaliar a percepção do estado de saúde dos idosos da Região Metropolitana de Belo Horizonte a partir dos dados da Pesquisa de Amostra por Domicílio/MG. A amostra utilizada foi de 963 pessoas, dentre as quais 403 eram do sexo masculino. A idade dos idosos variou entre $60 \mathrm{e}$ 99 anos, sendo a média 69,4 anos. Dentre eles, apenas 10,7\% consideram o seu estado de saúde como "ruim" ou "muito ruim". 67,9\% dos entrevistados relataram receber aposentadoria ou pensão previdenciária pública. Em relação aos hábitos de vida saudável, apenas $25 \%$ dos idosos referiram praticar atividade física regularmente e $41,7 \%$ se identificaram como tabagistas. Foi averiguado, ainda, que a autopercepção do estado de saúde se apresenta pior nos idosos com doenças crônicas, baixa escolaridade, menor ocupação laboral e baixa prática de atividade física.

Antes et al. (2014) descreveram o perfil socioeconômico e a auto-percepção de saúde da população com 60 anos ou mais residente em Florianópolis, com base no levantamento dos resultados obtidos em dois estudos epidemiológicos: Perfil do Idoso e EpiFloripa Idoso. A comparação dos estudos revelou aumento da escolaridade, das aposentadorias e da aquisição de planos de saúde, além da redução do percentual de idosos trabalhando e dos pensionistas no período de sete anos. Sobre a situação econômica, percebeu-se o aumento na autopercepção de melhoria, em comparação a quando tinham 50 anos de idade. Por outro lado, a saúde autorreferida passou de boa para regular ou ruim, sendo que esse fato, segundo os autores, pode estar relacionado ao aumento da exigência dos indivíduos com sua própria saúde.

Já o estudo de Holz et al. (2013) objetivou conhecer a prevalência e os fatores de déficit cognitivo na população idosa de Bagé (RS). Os principais fatores associados a esse tipo de déficit foram: sexo feminino, indivíduos mais velhos, de raça negra, amarela, parda ou indígena, menor nível de escolaridade, classe econômica baixa, sem aposentadoria, viver sem companheiro, presença de depressão e incapacidade instrumental da vida diária.

Celich et al. (2010) identificaram fatores que influenciam no envelhecimento com qualidade de vida, de acordo com a percepção de idosos participantes de grupos de terceira idade no município de Erechim (RS). Dentre as constatações, estão: o recebimento de aposentadoria, os relacionamentos familiares e sociais, a saúde física e mental, a liberdade de gerenciar a vida, a espiritualidade e as políticas governamentais. As narrativas das participantes ratificaram, também, a essencialidade da aposentadoria, enquanto benefício previdenciário, e da saúde como fatores que possibilitam o alcance de outros aspectos relacionados à qualidade de vida.

A pesquisa de Trentini et al. (2005) evidenciou que as principais situações desfavoráveis vivenciadas por idosos com doenças crônicas são as perdas e os danos causados por elas. Já entre as situações consideradas favoráveis, estão a aquisição de experiências e conhecimentos, bem como a aposentadoria, que é vista como liberação da rotina de trabalho. Por outro lado, alguns participantes manifestaram que o envolvimento laboral se caracterizou como um meio para o enfrentamento das adversidades transcorridas, assim como o cuidado consigo e com o corpo.

Andrade et al. (2010) pesquisaram as vivências de idosos soropositivos inscritos em uma unidade de referência do SUS de Belém (PA). Visualizou-se que o impacto do diagnóstico da doença abalou a afetividade dos sujeitos, seus laços de família e de amizade, bem como sua autoimagem. Diante disso, os indivíduos buscaram redes de apoio que pudessem contribuir com a situação de crise. Nesse contexto, o diagnóstico ocasionou, inclusive, a perda de projetos de futuro, tornando-se uma barreira na construção dos seus sonhos. Já a aposentadoria, em alguns casos, foi representada como algo benéfico e que mudou a dinâmica de vida, oportunizando outras vivências em função do tempo livre. 
Marin et al. (2013) investigaram as condições de saúde de pessoas com deficiência acima dos 50 anos e de seus cuidadores. Entre os participantes, $90,7 \%$ pertenciam a classe social baixa, sendo considerados pobres ou muito pobres. Observou-se que o principal provento era a aposentadoria, o que nem sempre garante os recursos necessários para a sobrevivência e para o atendimento das demandas de saúde. Sobre os cuidadores, ressalta-se que, em maior parcela, tinham acima de 60 anos, eram familiares da pessoa com deficiência, principalmente irmãos ou sobrinhos, e também relataram ter problemas de saúde.

França et al. (2013) descreveram o desenvolvimento de uma intervenção breve na preparação para a aposentadoria. Essa intervenção foi uma sessão única realizada em grupo, com três horas de duração, seguida de três monitoramentos. Tal atividade foi percebida pelos participantes como favorecedora de emoções positivas, obtenção de novos saberes e intercâmbio com outros sujeitos que vivenciavam experiências semelhantes. Na atividade foram abordados diferentes temas, dentre os quais está a necessidade de cuidados com a saúde física e emocional. Os autores apontam que esse tipo de atividade se configura como uma prática relevante para a prevenção e promoção de saúde, sobretudo em fases tardias do desenvolvimento.

Cavalcante e Freitas (2007) relatam a experiência de um trabalho com idosos cadastrados na Estratégia de Saúde da Família de Pacatuba (CE). As atividades caracterizaram-se como interdisciplinares, envolvendo os diferentes profissionais que atuam naquele local, oportunizando, assim, uma experiência integradora também para os usuários. No transcorrer das atividades foram realizados debates e ações sobre diversos temas, entre os quais: saúde física, estatuto do idoso, aposentadoria e práticas de reflexologia. Cabe enfatizar que, segundo os autores, o próprio grupo buscou e convidou novos integrantes com intuito de afastá-los do isolamento social e ocupar o tempo ocioso em virtude da aposentadoria.

\section{Aposentadoria e saúde do trabalhador}

Nesta categoria estão integrados 5 artigos. Miranda et al. (2009) analisaram a aposentadoria por invalidez dos servidores da Universidade Federal do Rio Grande do Norte, no período de 2000 a 2005, com diagnósticos de transtornos mentais e comportamentais. De um total de 43 aposentadorias, foi verificado que $58 \%$ eram de homens, $41 \%$ dos sujeitos tinham entre $41-50$ anos e $44 \%$ ocupavam cargo/função de nível médio. Nesse contexto, os diagnósticos foram: $61 \%$ apresentavam transtorno do humor; $19 \%$, transtorno do pensamento; $11 \%$, transtornos mentais orgânicos; e 9\%, transtornos de personalidade. Os autores discutem que, independente da história pregressa, o ambiente de trabalho dos cargos de nível médio possui um componente precipitador de manifestações psicopatológicas, o que pode estar relacionado ao baixo controle do trabalhador em relação ao seu processo de trabalho mediante à hierarquização da IES.

Brites, Abreu e Pinto (2014) descreveram as causas da morbidade dos servidores aposentados por invalidez em uma universidade pública. O estudo abrangeu 553 trabalhadores que se aposentaram nessa condição, dentre os quais $61,2 \%$ eram mulheres e $38,8 \%$ eram homens, sendo que o alcoolismo foi responsável por, respectivamente, $14 \%$ e $86 \%$ dos casos. Além desta, outras causas principais foram os transtornos mentais, neoplasias e doenças do sistema circulatório. Novamente, foi possível constatar a predominância de indivíduos com idades entre 30 e 60 anos, ou seja, aposentadorias que ocorreram entre o início e o auge da vida adulta. Analisa-se a relevância de reconhecer os fatores precipitadores de aposentadorias por invalidez, posto que isso possibilita a implementação de intervenções das políticas de promoção e proteção à saúde do trabalhador, levando-se em conta a realidade de cada organização e as demandas dos indivíduos que nela estão inseridos.

A investigação realizada por Brant e Gomes (2005), ancorada na perspectiva freudiana, buscou definir o sofrimento manifestado no ambiente de trabalho e seus destinos, cuja pesquisa foi realizada em uma organização em processo de reestruturação. Foi identificada a relação entre gestão do trabalho e sofrimento, sendo que o adoecimento é a expressão desse processo. Nesse sentido, a aposentadoria por invalidez, assim como a licença médica, se apresentou como manifestação que resulta de tal relação. Em relação especificamente à aposentadoria por invalidez, observou-se que ela abarcou representações que estavam relacionadas à forma como o indivíduo percebia seu trabalho, sendo entendida como recompensa e libertação do sofrimento do trabalho e das exigências da organização. Apesar disso, com o passar do tempo, em alguns casos ocorreram episódios depressivos e prevaleceu o sentimento de invalidez.

Finazzi-Santos e Siqueira (2011) apresentam um estudo de caso com base no óbito de um trabalhador bancário, visando avaliar se o trabalho poderia ser um dos fatores relacionado à decisão dele cometer suicídio. A história do protagonista foi construída com intensa dedicação ao trabalho, privilegiando esse contexto se 
comparado à família. Segundo os dados, ele estava a um ano de se aposentar e esse período da sua vida representava justamente a possibilidade de dar maior atenção às relações familiares. Contudo, nessa época, sua esposa pediu divórcio, e, somado a isso, seu perfil workaholic e as dificuldades na organização em que atuava foram elementos que geraram intensa desorganização em sua vida. Os autores questionam "o silêncio institucional" frente à proximidade de aposentadoria desse indivíduo, cujo envolvimento e dedicação ao trabalho eram intensos e, mesmo assim, não haviam políticas de recursos humanos que atendessem às suas demandas. Esse caso chama a atenção, sobretudo, para a necessidade de preparação em relação à aposentadoria, constituindo-se um desafio para a área de gestão de pessoas, especialmente nas práticas que visam o bem-estar dos trabalhadores.

A pesquisa de Garrido et al. (2007), por sua vez, se propôs a analisar o efeito do processo de estigmatização e discriminação no ambiente de trabalho sobre os cuidados cotidianos à saúde e ao bem-estar de homens vivendo com HIV/AIDS. Nela, averiguou-se que, dos 17 participantes, 14 estavam aposentados por invalidez ou recebiam auxílio doença, cuja condição era geradora de conflitos e exclusão, afetando a autoestima dos indivíduos. Algumas narrativas demonstraram que o conflito se dava entre estar "aposentado por invalidez" e perceber-se, de fato, como "inválido", posto que nem todos estavam incapacitados para o trabalho e/ou impossibilitados de produzir devido à doença. Isso também foi observado entre os que permaneceram desenvolvendo suas atividades laborais e precisavam se ausentar para realizar consultas ou em virtude das reações dos medicamentos retrovirais.

\section{Saúde do idoso e saúde do trabalhador}

Nesta categoria foram congregados três artigos que buscam discutir especificamente a interface entre saúde do idoso e saúde do trabalhador. Rezende e Dias (2007) identificaram a percepção de cuidadores familiares de idosos do território de uma Unidade Básica de Saúde de Betim-MG acerca do suporte recebido para o desenvolvimento de suas atividades. Esse estudo mostra o impacto negativo do cotidiano de trabalho dos cuidadores sobre seus aspectos gerais de saúde, que envolvem desde cansaço físico até depressão. Os participantes evidenciaram que se sentem solitários no desempenho de suas atividades e carecem de maior apoio por parte dos familiares dos idosos e dos profissionais da saúde.

O estudo de Tavares et al. (2010) objetivou conhecer o significado de ser idoso e identificar os fatores de prazer e sofrimento no cuidado aos idosos hospitalizados por parte dos profissionais de enfermagem. Evidenciouse que esses trabalhadores percebem o processo de envelhecimento relacionado às transformações da imagem corporal provocada pela velhice e adoecimento. O sofrimento dos profissionais estava pautado, principalmente, pelas situações em que houve a projeção de algum ente querido com a figura do idoso hospitalizado, o abandono do idoso pelos seus familiares e a iminência da morte do paciente. Por sua vez, as ocasiões que permitiam a interação entre idoso e profissional, quando havia motivação do paciente e este oferecia feedback positivo do trabalho desenvolvido, foram descritas como sendo satisfatórias.

Por fim, a investigação de Robazzi et al. (2009) buscou caracterizar os acidentes de trabalho sofridos por idosos, a partir dos registros em prontuários de uma unidade de emergência. Averiguou-se que um número significativo dos idosos que haviam sofrido acidente de trabalho descreveu-se aposentado, o que indica que esse público não necessariamente deixa de desempenhar atividades laborais após conquistar o recebimento de benefício previdenciário. Além disso, sobressaíram-se acidentes ocorridos no desempenho de tarefas no contexto doméstico e rural. Em mais de $90 \%$ dos casos, tais situações ocorreram em função de queda, sendo esse um elemento que merece destaque devido às repercussões que pode conferir ao idoso.

\section{DISCUSSÃO}

Mediante os resultados obtidos com o levantamento realizado para esta revisão, constatou-se que há um número escasso de publicações científicas no cenário brasileiro sobre os temas aposentadoria, saúde do idoso e saúde do trabalhador. Tanto o resultado total quanto o relativo a cada estratégia utilizada para a busca da produção sinalizam que tais temas apresentam uma produção restrita e que parece não condizer diretamente com o processo de envelhecimento populacional que, em certa medida, exige respostas importantes e imediatas.

Sobre as características metodológicas dos artigos analisados, percebeu-se a prevalência de estudos de natureza qualitativa, com o uso de entrevistas semiestruturadas, os quais atendem à demanda de entendimento da singularidade e dos significados que emergem nessa etapa da vida. Entretanto, sugere-se que novos estudos 
sejam executados, trazendo um diálogo com as abordagens quantitativas, possibilitando a mensuração dos aspectos concernentes à relação entre os temas de interesse e o aprofundamento dos mesmos.

Quanto às categorias temáticas apresentadas, de um modo geral, foi possível identificar diferentes fatores que implicam claramente sobre as condições de saúde de trabalhadores, idosos e aposentados, dentre os quais estão questões de natureza socioeconômica, étnico-racial e de gênero. Destaca-se a necessária discussão acerca das condições efetivas de trabalho, fundamentadas em políticas e práticas de gestão que promovem contextos pouco saudáveis para o sujeito, as quais podem ocasionar adoecimento, afastamento da atividade laboral e, inclusive, configurar a aposentadoria por invalidez. Tais questões podem impactar diretamente a qualidade de vida de trabalhadores ativos e inativos, assim como o modo pelo qual a aposentadoria é concebida e efetivada, gerando condições que potencialmente dificultam a adaptação e o enfrentamento ao período (Zanelli et al., 2010). Soma-se a isso o lugar do aposentado e do idoso diante do modelo capitalista que rege a atual estrutura societária, o qual ocasiona subvalorização e marginalização desses sujeitos, uma vez que supostamente estão distantes do principal meio de produção, que é o trabalho (Mendes et al., 2005).

Além disso, cabe ressaltar a posição que as redes de apoio social ocupam mediante essas situações da vida, tendo sido apontadas em uma quantidade significativa dos estudos analisados, estando relacionadas, especialmente, à manutenção de vínculos afetivos, convivência e proteção. Tais elementos são primordiais para o bem-estar na vida idosa, visto que a participação ativa em espaços familiares e comunitários carece de ser constantemente exercitada e aprimorada, o que pode promover o rompimento com o formato linear e estigmatizador - focado nas perdas e na aproximação com a morte - por meio do qual os sujeitos em fases tardias da vida foram compreendidos no decorrer da história (Neri \& Freire, 2000). Visualiza-se, portanto, a relevância da construção e adoção de referenciais teóricos e técnicos adequados para a compreensão e intervenção acerca de demandas multifacetadas que compõem a realidade, dentre as quais está o fenômeno do envelhecimento.

Averiguou-se, também, que os trabalhos analisados não discutem o fenômeno da aposentadoria de forma ampliada, no sentido de considerar a complexidade de fatores psicológicos, sociais e culturais que nele estão envolvidos. Percebeu-se uma ênfase nas questões de cunho econômico, enfatizando o benefício previdenciário em vez da inter-relação dos processos objetivos e subjetivos que coexistem nessa experiência. Chama a atenção o fato de que foi localizada apenas uma produção que parte dos estudos de aposentadoria para discutir aspectos relacionados à saúde, o que permite questionar se os trabalhos nessa área têm marginalizado, em certa medida, o diálogo com a perspectiva da saúde, privilegiando outros aspectos.

Problematiza-se essas constatações, visto que o fenômeno da aposentadoria precisa ser pensado face às diversas condições que podem estar sobrepostas em sua vivência, em uma perspectiva multidimensional e longitudinal, para a qual os indivíduos precisam ser educados ao longo de todo o seu ciclo vital (França \& Soares, 2009). Dessa forma, aponta-se que o planejamento para esse período deve ocorrer integrado às particularidades do contexto de vida de cada trabalhador, considerando a rede de seus vínculos laborais, afetivos e sociais.

O conjunto de elementos discutidos constitui-se importante para a formulação e implementação de políticas de saúde para a população brasileira. Ressalta-se que as peculiaridades identificadas podem contribuir, sobretudo, no planejamento e execução de ações de promoção da saúde para os públicos de idosos e aposentados, pois possibilitam analisar alguns Determinantes Sociais de Saúde a partir da heterogeneidade de situações que estão envolvidas nos processos de trabalho e do desenvolvimento em fases tardias. Cabe enfatizar que tais elementos precisam ser alvo de reflexão e discussão tanto na elaboração de políticas públicas que atendam à população de idosos e aposentados como na formação de profissionais que atuam com tais grupos em diferentes espaços sociais.

Por fim, levando-se em conta as possíveis limitações decorrentes das bases de dados consultadas e dos critérios de inclusão e de exclusão especificados, enfatiza-se que esta revisão permitiu descrever a produção científica sobre aposentadoria, saúde do idoso e saúde do trabalhador, contribuindo para dar visibilidade aos assuntos e a demanda de que novos estudos diretamente relacionados às interfaces entre os mesmos possam ser desenvolvidos, buscando suprir lacunas. Acredita-se que é importante dar continuidade a essa análise, por meio de outras revisões, ampliando o lócus de busca e a possibilidade de acompanhar e comparar resultados. Sugere-se aos profissionais da saúde, especialmente aos psicólogos, maior atuação tanto na intervenção quanto na investigação científica, considerando-se as inter-relações entre os temas, uma vez que tais aspectos podem repercutir diretamente na qualidade de vida dos indivíduos em diferentes contextos, sobretudo, nas organizações de trabalho. 


\section{REFERÊNCIAS}

Andrade, H. A. S., Silva, S. K., \& Santos, M. I. P. O. (2010). AIDS em idosos: Vivências dos doentes. Escola Anna Nery Revista de Enfermagem, 14(4), 712-719. doi: http://dx.doi.org/10.1590/S1414-81452010000400009

Antes, D. L., Ribeiro, D. F., Schneider, I. J. C., Benedetti, T. R. B., \& D’orsi, E. (2014). Perfil socioeconômico dos idosos de Florianópolis: Análise comparativa dos estudos Perfil do Idoso 2002 e EpiFloripa Idoso 2009. Revista Brasileira de Epidemiologia, 17(1), 189-202. doi: http://dx.doi.org/10.1590/1415-790X201400010015ENG

Antunes, M. H., \& Moré, C. L. O. O. (2014). Família, trabalho e aposentadoria: Uma revisão da produção científica no cenário brasileiro. Contextos Clínicos, 7(2), 145-154.

Antunes, M. H., Soares, D. H. P., \& Silva, N. (2015). Orientação para aposentadoria nas organizações: Histórico, gestão de pessoas e indicadores para uma possível associação com a gestão do conhecimento. Perspectivas em Gestão \& Conhecimento, 5(1), 43-63.

Beyea, S. C., \& Nicoll, L. H. (1998). Writing an integrative review. AORN Journal, 67, 877-880. doi: http://dx.doi.org/10.1016/ S0001-2092(06)62653-7

Brasil. (2006). Política Nacional de Promoção da Saúde. Brasília: MS. Recuperado de http://bvsms.saude.gov.br/bvs/publicacoes/ politica_nacional_promocao_saude_3ed.pdf

Brasil. (2014). Manual de enfrentamento à violência contra a pessoa idosa. Brasília: SDH Recuperado de http://www.sdh.gov.br/ assuntos/pessoa-idosa/publicacoes/violencia-contra-a-pessoa-idosa

Brant, L. C., \& Gomez, C. M. (2005). O sofrimento e seus destinos na gestão do trabalho. Ciência e saúde coletiva, 10(4), 939952. doi: http://dx.doi.org/10.1590/S1413-81232005000400017

Brites, R. M. R., Abreu, Â. M. M., \& Pinto, J. E. S. S. (2014). Prevalência de alcoolismo no perfil das aposentadorias por invalidez dentre trabalhadores de uma universidade federal. Revista Brasileira de Enfermagem, 67(3), 373-380. doi: 10.5935/00347167.20140049

Carvalho, F. F., Santos, J. N., Souza, L. M., \& Souza, N. R. M. (2012). Análise da percepção do estado de saúde dos idosos da região metropolitana de Belo Horizonte. Revista Brasileira de Geriatria e Gerontologia, 15(2), 285-294. doi: http://dx.doi. org/10.1590/S1809-98232012000200011

Cavalcante, P. M. T., \& Freitas, M. C. (2007). Em busca de um viver saudável: Relato de experiência com um grupo de idosos cadastrados na estratégia saúde da família de Pacatuba - CE. Revista da Rede de Enfermagem do Nordeste, 8(1), 92-99.

Celich, K. L. S., Creutzberg, M., Goldim, J. R., \& Gomes, I. (2010). Envelhecimento com qualidade de vida: A percepção de idosos participantes de grupos de terceira idade. Revista Mineira de Enfermagem, 14(2), 226-232.

Cockell, F. F. (2014). Idosos aposentados no mercado de trabalho informal: Trajetórias ocupacionais na construção civil. Psicologia \& Sociedade, 26(2), 461-471. doi: 10.1590/S0102-71822014000200022

Denton, F. T., \& Spencer, B. G. (2009). What is retirement? A review and assessment of alternative concepts and measures. Canadian Journal on Aging, 28(1), 63-76. doi: http://dx.doi.org/10.1017/S0714980809090047

Finazzi-Santos, M. A., \& Siqueira, M. V. S. (2011). Considerações sobre trabalho e suicídio: Um estudo de caso. Revista Brasileira de Saúde Ocupacional, 36(123), 71-83.

França, C. L. (2014a). Aposentadoria: Crise ou liberdade? In S. G. Murta, C. Leandro-França \& Seidl, J. Programas de educação para aposentadoria: Como planejar, implementar e avaliar (pp. 54-65). Novo Hamburgo: Sinopsys.

França, C. L. (2014b). Prevenção e promoção da saúde mental, políticas públicas sobre envelhecimento ativo e educação para aposentadoria. In S. G. Murta, C. Leandro-França \& Seidl, J. Programas de educação para aposentadoria: Como planejar, implementar e avaliar (pp. 22-36). Novo Hamburgo: Sinopsys.

França, C. L., Murta, S. G., Negreiros, J. L., Pedralho, M., \& Carvalhedo, R. (2013). Intervenção breve na preparação para aposentadoria. Revista Brasileira de Orientação Profissional, 14(1), 99-110.

França, L. H. F. P., \& Soares, D. H. P. (2009). Preparação para a aposentadoria como parte da educação ao longo da vida. Psicologia: Ciência e Profissão, 29(4) 738-751.

Garrido, P. B., Paiva, V., Nascimento, V. L. V., Sousa, J. B., \& Santos, N. J. S. (2007). AIDS, estigma e desemprego: Implicações para os serviços de saúde. Revista de Saúde Pública, 41(1), 72-79. doi: http://dx.doi.org/10.1590/S003489102007000900012

Holz, A. W., Nunes, B. P., Thumé, E., Lange, C., \& Facchini, L. A. (2013). Prevalence of cognitive impairment and associated factors among the elderly in Bagé, Rio Grande do Sul, Brazil. Revista Brasileira de Epidemiologia, 16(4), 880-888. doi: http:// dx.doi.org/10.1590/S1415-790X2013000400008 
Instituto Brasileiro de Geografia e Estatística. (2011). Sinopse do censo demográfico 2010.. Rio de Janeiro: IBGE. Recuperado de http://www.censo2010.ibge.gov.br/sinopse/webservice/

Johnson, R. W. (2009). Family, public policy, and retirement decisions: Introduction to the special issue. Research on Aging, 31(2), 139-152. doi: 10.1177/0164027508328307

Lei no 8.842, de 4 de janeiro de 1994 (1994, 04 de janeiro). Dispõe sobre a Política Nacional do Idoso, cria o Conselho Nacional do Idoso e dá outras providências. Recuperado de http://www.planalto.gov.br/ccivil_03/Leis/L8842.htm

Lei no 10.741, de 1ํo de outubro de 2003 (2003, 1 de outubro). Dispõe sobre o Estatuto do Idoso e dá outras providências. Recuperado de http://www.planalto.gov.br/ccivil_03/Leis/2003/L10.741.htm

Lesbaupin, S. F., \& Malerbi, F. (2006). O idoso por ele mesmo. Revista Kairós, 9(2), 51-67.

Marin, M. J. S., Lorenzetti, D., Chacon, M. C. M., Polo, M. C., Martins, V. S., \& Moreira, S. A. C. (2013). As condições de vida e saúde de pessoas acima de 50 anos com deficiência e seus cuidadores em um município paulista. Revista Brasileira de Geriatria e Gerontologia, 16(2), 365-374. doi: http://dx.doi.org/10.1590/S1809-98232013000200016

Mendes, K. D. S., Silveira, R. C. C. P., \& Galvão, C. M. (2008). Revisão integrativa: Método de pesquisa para incorporação de evidências na saúde e na enfermagem. Texto Contexto - Enfermagem, 17(4), 758-764.

Mendes, M. R. S. S. B., Gusmão, J. L., Faro, A. C. M., \& Leite, R. C. B. O. (2005). A situação social do idoso no Brasil: Uma breve consideração. Acta Paulista de Enfermagem, 18(4), 422-426.

Miranda, F. A. N., Carvalho, G. R. P., Fernandes, R. L., Silva, M. B., \& Sabino, M. G. G. (2009). Saúde mental, trabalho e aposentadoria: Focalizando a alienação mental. Revista Brasileira de Enfermagem, 62(5), 711-716. doi: http://dx.doi. org/10.1590/S0034-71672009000500010

Neri, A. L., \& Freire, A. S. (2000). E por falar em boa velhice. São Paulo: Papirus.

Olabuénaga, J. I. R. (2009). Metodología de la investigación cualiatitativa. Bilbao: Universidad de Deusto.

Organizações das Nações Unidas. (2002). Plano internacional de ação mundial para o envelhecimento. Madrid: ONU. Recuperado de http://www.un.org/en/events/pastevents/pdfs/Madrid_plan.pdf

Panozzo, E. A. L., \& Monteiro, J. K. (2013). Aposentadoria e saúde mental: Uma revisão de literatura. Cadernos de Psicologia Social do Trabalho, 16(2), 199-209.

Paskulin, L. M. G., \& Vianna, L. A. C. (2007). Perfil sociodemográfico e condições de saúde auto referidas de idosos de Porto Alegre. Revista de Saúde Pública, 41(5), 757-768. doi: http://dx.doi.org/10.1590/S0034-89102007000500010

Rezende, M. C. F., \& Dias, E. C. (2007). Os cuidadores familiares de idosos: Um novo agente de saúde no SUS sob o olhar da saúde do trabalhador. Revista Médica de Minas Gerais, 17(1/2, supl.4), 288-293.

Robazzi, M. L. C. C., Marziale, M. H. P., Rodrigues, R. A. P., Silveira, C. A., \& Alves, L. A. (2009). Acidentes e agravos à saúde dos idosos nos ambientes de trabalho, Revista Enfermagem UERJ, 17(3), 309-314.

Rodrigues, C. L. (2001). Homem de pijama: O imaginário masculino em relação à aposentadoria. Revista Kairós, 4(2), 69-82.

Salimene, A. C. M., Oliveira, B., Mussolini, C. C., Novembre, R., Zagabria, D., Custódio, I... Bianco, V. (2006). Perfil previdenciário de idosos internados em um hospital da rede pública da cidade de São Paulo. Revista Kairós, 9(1), 197-220.

Santos, M. F. S. (1990). Identidade e aposentadoria. São Paulo: EPU.

Soares, D. H. P., \& Costa, A. B. (2011). Aposent-Ação: Aposentadoria para ação. São Paulo: Vetor.

Szinovacz, M. E., Ekerdt, D. J., Butt, A., Barton, K., \& Oala, C. R. (2012). Families and retirement. In R. Blieszner \& V. H. Bedford (Eds.), Handbook of families and aging (pp. 461-488). Califórnia: ABC- CLIO LLC.

Tavares, J. P., Beck, C. L. C., Silva, R. M., Beuter, M., Prestes, F. C., \& Rocha, L. (2010). Prazer e sofrimento de trabalhadoras de enfermagem que cuidam de idosos hospitalizados. Escola Anna Nery Revista de Enfermagem, 14(2), 253-259. doi: http://dx.doi.org/10.1590/S1414-81452010000200007

Trentini, M., Silva, S. H., Valle, M. L., \& Hammerschmidt, K. S. A. (2005). Enfrentamento de situações adversas e favoráveis por pessoas idosas em condições crônicas de saúde. Revista Latino-Americana de Enfermagem, 13(1), 38-45. doi: http://dx.doi. org/10.1590/S0104-11692005000100007

Zanelli, J. C., Silva, N., \& Soares, D. H. P. (2010). Orientação para aposentadoria nas organizações de trabalho: Construção de projetos para o pós-carreira. Porto Alegre: Artmed. 